Vladimir G. Maz'ya

Tatyana O. Shaposhnikova

\title{
Theory of Sobolev Multipliers
}

With Applications to Differential and Integral Operators

照 Springer 


\section{Contents}

Introduction $\ldots \ldots \ldots \ldots \ldots \ldots \ldots \ldots \ldots \ldots \ldots \ldots \ldots$

\section{Part I Description and Properties of Multipliers}

1 Trace Inequalities for Functions in Sobolev Spaces ........ 7

1.1 Trace Inequalities for Functions in $w_{1}^{m}$ and $W_{1}^{m} \ldots \ldots \ldots \ldots, 7$

1.1.1 The Case $m=1 \ldots \ldots \ldots \ldots \ldots \ldots \ldots \ldots \ldots, 7$

1.1 .2 The Case $m \geq 1 \ldots \ldots \ldots \ldots \ldots \ldots \ldots \ldots \ldots \ldots \ldots$

1.2 Trace Inequalities for Functions in $w_{p}^{m}$ and $W_{p}^{m}, p>1 \ldots \ldots 14$

1.2 .1 Preliminaries ............................. 14

1.2 .2 The $(p, m)$-Capacity $\ldots \ldots \ldots \ldots \ldots \ldots \ldots \ldots \ldots \ldots$

1.2.3 Estimate for the Integral of Capacity of a Set Bounded by a Level Surface ....................... 19

1.2.4 Estimates for Constants in Trace Inequalities ....... 22

1.2.5 Other Criteria for the Trace Inequality (1.2.29)

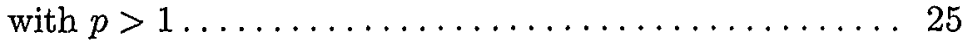

1.2.6 The Fefferman and Phong Sufficient Condition ....... 28

1.3 Estimate for the $L_{q}$-Norm with respect to an Arbitrary

Measure................................. 29

1.3.1 The case $1 \leq p<q \ldots \ldots \ldots \ldots \ldots \ldots \ldots \ldots \ldots, \ldots \ldots$

1.3.2 The case $q<p \leq n / m \ldots \ldots \ldots \ldots \ldots \ldots \ldots \ldots$

2 Multipliers in Pairs of Sobolev Spaces .............. 33

2.1 Introduction .............................. 33

2.2 Characterization of the Space $M\left(W_{1}^{m} \rightarrow W_{1}^{l}\right) \ldots \ldots \ldots \ldots \ldots 35$

2.3 Characterization of the Space $M\left(W_{p}^{m} \rightarrow W_{p}^{l}\right)$ for $p>1 \ldots \ldots 38$

2.3.1 Another Characterization of the Space $M\left(W_{p}^{m} \rightarrow W_{p}^{l}\right)$ for $0<l<m, p m \leq n, p>1 \ldots \ldots \ldots \ldots \ldots \ldots 43$

2.3.2 Characterization of the Space $M\left(W_{p}^{m} \rightarrow W_{p}^{l}\right)$ for $p m>n, p>1 \ldots \ldots \ldots \ldots \ldots \ldots \ldots \ldots$ 
2.3.3 One-Sided Estimates for Norms of Multipliers in the Case $p m \leq n \ldots \ldots \ldots \ldots \ldots \ldots \ldots \ldots$

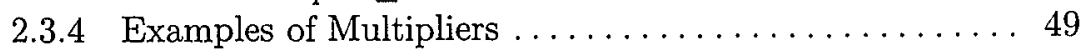

2.4 The Space $M\left(W_{p}^{m}\left(\mathbb{R}_{+}^{n}\right) \rightarrow W_{p}^{l}\left(\mathbb{R}_{+}^{n}\right)\right) \ldots \ldots \ldots \ldots \ldots \ldots \ldots$

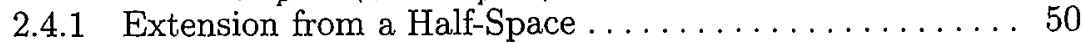

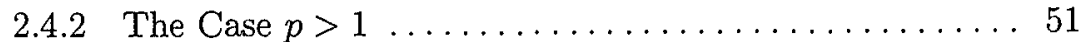

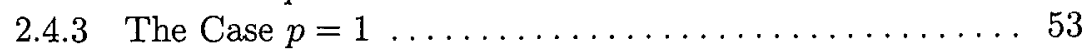

2.5 The Space $M\left(W_{p}^{m} \rightarrow W_{p}^{-k}\right) \ldots \ldots \ldots \ldots \ldots \ldots \ldots \ldots \ldots \ldots$

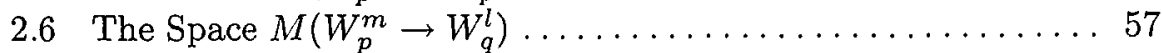

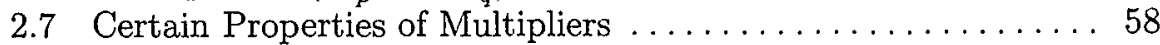

2.8 The Space $M\left(w_{p}^{m} \rightarrow w_{p}^{l}\right) \ldots \ldots \ldots \ldots \ldots \ldots \ldots \ldots \ldots \ldots \ldots 60 \ldots \ldots$

2.9 Multipliers in Spaces of Functions with Bounded Variation.... 63

2.9.1 The Spaces $M b v$ and $M B V \ldots \ldots \ldots \ldots \ldots \ldots \ldots \ldots \ldots \ldots \ldots$

3 Multipliers in Pairs of Potential Spaces ............... 69

3.1 Trace Inequality for Bessel and Riesz Potential Spaces . . . . . 69

3.1.1 Properties of Bessel Potential Spaces . . . . . . . . . . 70

3.1.2 Properties of the $(p, m)$-Capacity $\ldots \ldots \ldots \ldots \ldots \ldots \ldots 71$

3.1 .3 Main Result ...................... 73

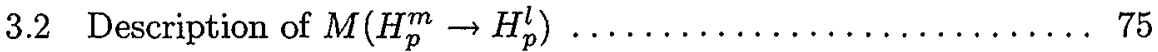

3.2.1 Auxiliary Assertions . . . . . . . . . . . . . 75

3.2.2 Imbedding of $M\left(H_{p}^{m} \rightarrow H_{p}^{l}\right)$ into $M\left(H_{p}^{m-l} \rightarrow L_{p}\right) \ldots 76$

3.2.3 Estimates for Derivatives of a Multiplier.......... 78

3.2.4 Multiplicative Inequality for the Strichartz Function . . 79

3.2.5 Auxiliary Properties of the Bessel Kernel $G_{l} \ldots \ldots \ldots 80$

3.2.6 Upper Bound for the Norm of a Multiplier... . . . . . . 81

3.2.7 Lower Bound for the Norm of a Multiplier . . . . . . . . . 85

3.2.8 Description of the Space $M\left(H_{p}^{m} \rightarrow H_{p}^{l}\right) \ldots \ldots \ldots \ldots$

3.2.9 Equivalent Norm in $M\left(H_{p}^{m} \rightarrow H_{p}^{l}\right)$ Involving the Norm in $L_{m p /(m-l)} \ldots \ldots \ldots \ldots \ldots \ldots \ldots$

3.2.10 Characterization of $M\left(H_{p}^{m} \rightarrow H_{p}^{l}\right), m>l$, Involving the Norm in $L_{1, \text { unif } \ldots \ldots \ldots \ldots \ldots \ldots \ldots . \ldots \ldots}$

3.2.11 The Space $M\left(H_{p}^{m} \rightarrow H_{p}^{l}\right)$ for $m p>n \ldots \ldots \ldots \ldots$

3.3 One-Sided Estimates for the Norm in $M\left(H_{p}^{m} \rightarrow H_{p}^{l}\right) \ldots \ldots \ldots 95$

3.3.1 Lower Estimate for the Norm in $M\left(H_{p}^{m} \rightarrow H_{p}^{l}\right)$

Involving Morrey Type Norms . . . . . . . . . . . 96

3.3.2 Upper Estimate for the Norm in $M\left(H_{p}^{m} \rightarrow H_{p}^{l}\right)$

Involving Marcinkiewicz Type Norms ............ 96

3.3.3 Upper Estimates for the Norm in $M\left(H_{p}^{m} \rightarrow H_{p}^{l}\right)$ Involving Norms in $H_{n / m}^{l} \ldots \ldots \ldots \ldots \ldots \ldots$

3.4 Upper Estimates for the Norm in $M\left(H_{p}^{m} \rightarrow H_{p}^{l}\right)$ by Norms in Besov Spaces ........................ 99

3.4 .1 Auxiliary Assertions . . . . . . . . . . . . . . . . . 99

3.4 .2 Properties of the Space $B_{q, \infty}^{\mu} \ldots \ldots \ldots \ldots \ldots \ldots \ldots$ 
3.4.3 Estimates for the Norm in $M\left(H_{p}^{m} \rightarrow H_{p}^{l}\right)$ by the Norm in $B_{q, \infty}^{\mu} \ldots \ldots \ldots \ldots \ldots \ldots \ldots \ldots \ldots \ldots$

3.4.4 Estimate for the Norm of a Multiplier in $M H_{p}^{l}\left(\mathbb{R}^{1}\right)$ by the $q$-Variation ....................... 110

3.5 Miscellaneous Properties of Multipliers in $M\left(H_{p}^{m} \rightarrow H_{p}^{l}\right) \ldots 111$

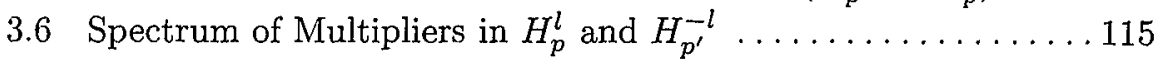

3.6.1 Preliminary Information................... 115

3.6.2 Facts from Nonlinear Potential Theory . . . . . . . . . 117

3.6.3 Main Theorem ........................... 118

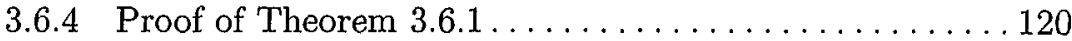

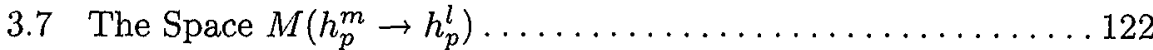

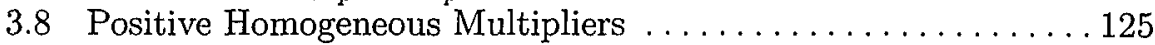

3.8.1 The Space $M\left(H_{p}^{m}\left(\partial \mathcal{B}_{1}\right) \rightarrow H_{p}^{l}\left(\partial \mathcal{B}_{1}\right)\right) \ldots \ldots \ldots \ldots \ldots . \ldots \ldots$

3.8.2 Other Normalizations of the Spaces $h_{p}^{m}$ and $H_{p}^{m} \ldots \ldots .127$

3.8.3 Positive Homogeneous Elements of the Spaces $M\left(h_{p}^{m} \rightarrow h_{p}^{l}\right)$ and $M\left(H_{p}^{m} \rightarrow H_{p}^{l}\right) \ldots \ldots \ldots \ldots \ldots \ldots 130$

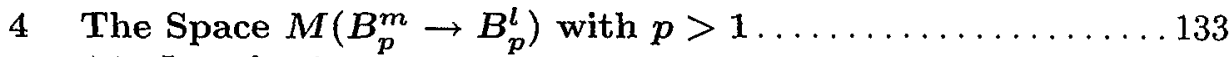

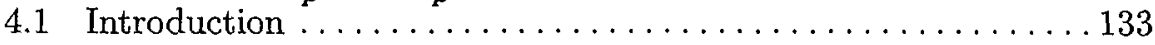

4.2 Properties of Besov Spaces....................... 134

4.2.1 Survey of Known Results ..................... 134

4.2.2 Properties of the Operators $\mathfrak{D}_{p, l}$ and $D_{p, l} \ldots \ldots \ldots 136$

4.2.3 Pointwise Estimate for Bessel Potentials . . . . . . . . . 138

4.3 Proof of Theorem 4.1.1 ......................... 141

4.3.1 Estimate for the Product of First Differences......... 141

4.3.2 Trace Inequality for $B_{p}^{k}, p>1 \ldots \ldots \ldots \ldots \ldots \ldots 143$

4.3.3 Auxiliary Assertions Concerning $M\left(B_{p}^{m} \rightarrow B_{p}^{l}\right) \ldots \ldots 145$

4.3.4 Lower Estimates for the Norm in $M\left(B_{p}^{m} \rightarrow B_{p}^{l}\right) \ldots \ldots 146$

4.3.5 Proof of Necessity in Theorem 4.1.1............. 149

4.3.6 Proof of Sufficiency in Theorem 4.1.1 .......... 155

4.3.7 The Case $m p>n \ldots \ldots \ldots \ldots \ldots \ldots \ldots \ldots \ldots$

4.3.8 Lower and Upper Estimates for the Norm

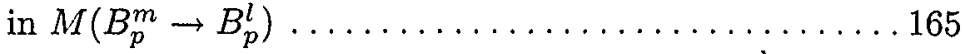

4.4 Sufficient Conditions for Inclusion into $M\left(W_{p}^{m} \rightarrow W_{p}^{l}\right)$

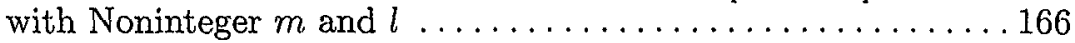

4.4.1 Conditions Involving the Space $B_{q, \infty}^{\mu} \ldots \ldots \ldots \ldots \ldots 166$

4.4.2 Conditions Involving the Fourier Transform ........ 168

4.4.3 Conditions Involving the Space $B_{q, p}^{l} \ldots \ldots \ldots \ldots \ldots 170$

4.5 Conditions Involving the Space $H_{n / m}^{l} \ldots \ldots \ldots \ldots \ldots \ldots \ldots \ldots \ldots \ldots \ldots \ldots$

4.6 Composition Operator on $M\left(W_{p}^{m} \rightarrow W_{p}^{l}\right) \ldots \ldots \ldots \ldots \ldots \ldots 174$ 


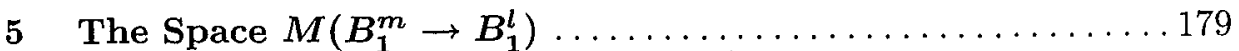

5.1 Trace Inequality for Functions in $B_{1}^{l}\left(\mathbb{R}^{n}\right) \ldots \ldots \ldots \ldots \ldots \ldots \ldots$

5.1 .1 Auxiliary Facts . . . . . . . . . . . . . . . . . . 180

$5.1 .2 \quad$ Main Result .......................... 183

5.2 Properties of Functions in the Space $B_{1}^{k}\left(\mathbb{R}^{n}\right) \ldots \ldots \ldots \ldots \ldots 185$

5.2.1 Trace and Imbedding Properties............... 185

5.2.2 Auxiliary Estimates for the Poisson Operator ......... 189

5.3 Descriptions of $M\left(B_{1}^{m} \rightarrow B_{1}^{l}\right)$ with Integer $l \ldots \ldots \ldots \ldots \ldots 193$

5.3.1 A Norm in $M\left(B_{1}^{m} \rightarrow B_{1}^{l}\right) \ldots \ldots \ldots \ldots \ldots \ldots \ldots \ldots . \ldots \ldots$

5.3.2 Description of $M\left(B_{1}^{m} \rightarrow B_{1}^{l}\right)$ Involving $\mathfrak{D}_{1, l} \ldots \ldots \ldots \ldots 199$

5.3.3 $M\left(B_{1}^{m}\left(\mathbb{R}^{n}\right) \rightarrow B_{1}^{l}\left(\mathbb{R}^{n}\right)\right)$ as the Space of Traces ......201

5.3.4 Interpolation Inequality for Multipliers ........... 202

5.4 Description of the Space $M\left(B_{1}^{m} \rightarrow B_{1}^{l}\right)$ with Noninteger $l \ldots 203$

5.5 Further Results on Multipliers in Besov and Other Function Spaces ...............................206

5.5.1 Peetre's Imbedding Theorem............... 206

5.5.2 Related Results on Multipliers in Besov and Triebel-Lizorkin Spaces.................208

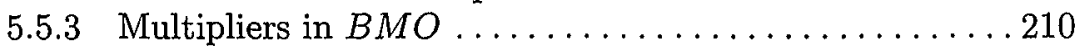

6 Maximal Algebras in Spaces of Multipliers . . . . . . . . . 213

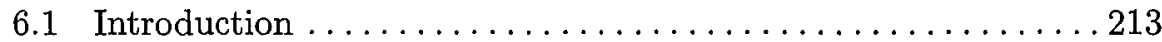

6.2 Pointwise Interpolation Inequalities for Derivatives......... 214

6.2.1 Inequalities Involving Derivatives of Integer Order . . . . 214

6.2.2 Inequalities Involving Derivatives of Fractional Order . . 215

6.3 Maximal Banach Algebra in $M\left(W_{p}^{m} \rightarrow W_{p}^{l}\right) \ldots \ldots \ldots \ldots \ldots 220$

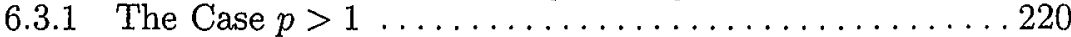

6.3.2 Maximal Banach Algebra in $M\left(W_{1}^{m} \rightarrow W_{1}^{l}\right) \ldots \ldots \ldots 224$

6.4 Maximal Algebra in Spaces of Bessel Potentials ........... 227

6.4.1 Pointwise Inequalities Involving the Strichartz

Function ...........................227

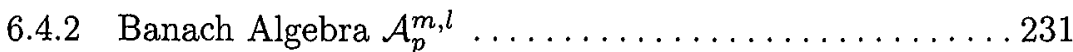

6.5 Imbeddings of Maximal Algebras . . . . . . . . . . . . . . . 233

7 Essential Norm and Compactness of Multipliers . . . . . . . . 241

7.1 Auxiliary Assertions . . . . . . . . . . . . . . . . . . . . . . . 243

7.2 Two-Sided Estimates for the Essential Norm. The Case

$m>l \ldots \ldots \ldots \ldots \ldots \ldots \ldots \ldots \ldots \ldots . \ldots . \ldots . \ldots . \ldots 248$

7.2.1 Estimates Involving Cutoff Functions . . . . . . . . 248

7.2.2 Estimate Involving Capacity (The Case

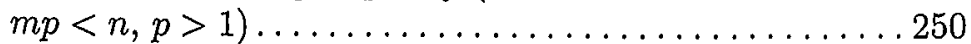

7.2.3 Estimates Involving Capacity (The Case

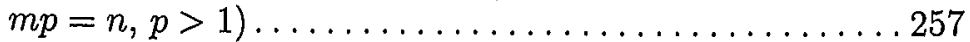

7.2.4 Proof of Theorem 7.0.3 ..................261

7.2.5 Sharpening of the Lower Bound for the Essential Norm in the Case $m>l, m p \leq n, p>1 \ldots \ldots \ldots 262$ 
7.2.6 Estimates of the Essential Norm for $m p>n, p>1$

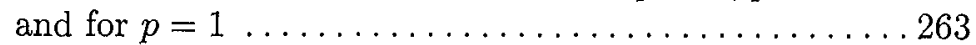

7.2.7 One-Sided Estimates for the Essential Norm . . . . . . . . 266

7.2.8 The Space of Compact Multipliers ............... 267

7.3 Two-Sided Estimates for the Essential Norm

in the Case $m=l \ldots \ldots \ldots \ldots \ldots \ldots \ldots \ldots \ldots . \ldots . \ldots . \ldots 270$

7.3.1 Estimate for the Maximum Modulus of a Multiplier in $W_{p}^{l}$ by its Essential Norm .................. 270

7.3.2 Estimates for the Essential Norm Involving Cutoff Functions (The Case $l p \leq n, p>1) \ldots \ldots \ldots \ldots \ldots 272$

7.3.3 Estimates for the Essential Norm Involving Capacity (The Case $l p \leq n, p>1$ ) ..................277

7.3.4 Two-Sided Estimates for the Essential Norm in the Cases $l p>n, p>1$, and $p=1 \ldots \ldots \ldots \ldots 278$

7.3.5 Essential Norm in $M W_{p}^{l} \ldots \ldots \ldots \ldots \ldots \ldots \ldots \ldots . \ldots \ldots 1$

8 Traces and Extensions of Multipliers.............. 285

8.1 Introduction .............................. 285

8.2 Multipliers in Pairs of Weighted Sobolev Spaces in $\mathbb{R}_{+}^{n} \ldots \ldots 285$

8.3 Characterization of $M\left(W_{p}^{t, \beta} \rightarrow W_{p}^{s, \alpha}\right) \ldots \ldots \ldots \ldots \ldots \ldots \ldots 288$

8.4 Auxiliary Estimates for an Extension Operator . . . . . . . . . . 292

8.4.1 Pointwise Estimates for $T \gamma$ and $\nabla T \gamma \ldots \ldots \ldots \ldots 292$

8.4.2 Weighted $L_{p}$-Estimates for $T \gamma$ and $\nabla T \gamma \ldots \ldots \ldots \ldots 294$

8.5 Trace Theorem for the Space $M\left(W_{p}^{t, \beta} \rightarrow W_{p}^{s, \alpha}\right) \ldots \ldots \ldots \ldots 297$

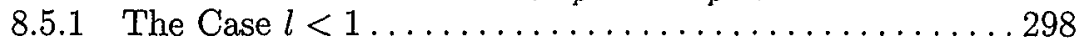

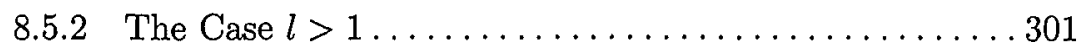

8.5.3 Proof of Theorem 8.5.1 for $l>1 \ldots \ldots \ldots \ldots \ldots 303$

8.6 Traces of Multipliers on the Smooth Boundary of a Domain. . . 304

8.7 $M W_{p}^{l}\left(\mathbb{R}^{n}\right)$ as the Space of Traces of Multipliers in the

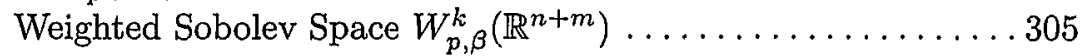

8.7.1 Preliminaries ............................. 305

8.7.2 A Property of Extension Operator .............. 306

8.7.3 Trace and Extension Theorem for Multipliers ........ 308

8.7.4 Extension of Multipliers from $\mathbb{R}^{n}$ to $\mathbb{R}_{+}^{n+1} \ldots \ldots \ldots 311$

8.7.5 Application to the First Boundary Value Problem

in a Half-Space. ............................ 311

8.8 Traces of Functions in $M W_{p}^{l}\left(\mathbb{R}^{n+m}\right)$ on $\mathbb{R}^{n} \ldots \ldots \ldots \ldots \ldots 312$

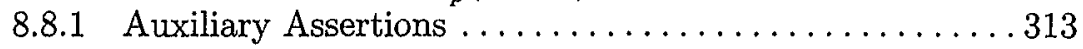

8.8.2 Trace and Extension Theorem ................ 315

8.9 Multipliers in the Space of Bessel Potentials as Traces of Multipliers ....................................... 319

8.9.1 Bessel Potentials as Traces ................... 319

8.9.2 An Auxiliary Estimate for the Extension Operator $\mathcal{T}$. . 320

8.9.3 $M H_{p}^{l}$ as a Space of Traces .................. 322 
9 Sobolev Multipliers in a Domain, Multiplier Mappings

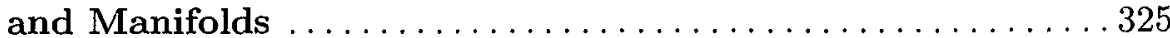

9.1 Multipliers in a Special Lipschitz Domain.............. 326

9.1.1 Special Lipschitz Domains . . . . . . . . . . . . . . . 326

9.1 .2 Auxiliary Assertions ......................... 326

9.1.3 Description of the Space of Multipliers ............ 329

9.2 Extension of Multipliers to the Complement of a Special

Lipschitz Domain............................... 332

9.3 Multipliers in a Bounded Domain ................ 336

9.3.1 Domains with Boundary in the Class $C^{0,1} \ldots \ldots \ldots 336$

9.3.2 Auxiliary Assertions ....................... 337

9.3.3 Description of Spaces of Multipliers in a Bounded

Domain with Boundary in the Class $C^{0,1} \ldots \ldots$. . . . 339

9.3.4 Essential Norm and Compact Multipliers

in a Bounded Lipschitz Domain . . . . . . . . . . . . . . 340

9.3.5 The Space $M L_{p}^{1}(\Omega)$ for an Arbitrary

Bounded Domain .......................... 346

9.4 Change of Variables in Norms of Sobolev Spaces........... 350

$9.4 .1(p, l)$-Diffeomorphisms...................... 350

9.4.2 More on $(p, l)$-Diffeomorphisms.................. 352

9.4.3 A Particular $(p, l)$-Diffeomorphism $\ldots \ldots \ldots \ldots \ldots \ldots 353$

$9.4 .4 \quad(p, l)$-Manifolds.............................. 356

9.4.5 Mappings $T_{p}^{m, l}$ of One Sobolev Space into Another . . . 357

9.5 Implicit Function Theorems. . . . . . . . . . . . . . . . . 364

9.6 The Space $M\left(\stackrel{\circ}{W}_{p}^{m}(\Omega) \rightarrow W_{p}^{l}(\Omega)\right) \ldots \ldots \ldots \ldots \ldots \ldots \ldots \ldots \ldots$

9.6.1 Auxiliary Results ......................... 367

9.6.2 Description of the Space $M\left(\mathscr{W}_{p}^{m}(\Omega) \rightarrow W_{p}^{l}(\Omega)\right) \ldots \ldots 369$

Part II Applications of Multipliers to Differential and Integral Operators

10 Differential Operators in Pairs of Sobolev Spaces . . . . . . . 373

10.1 The Norm of a Differential Operator: $W_{p}^{h} \rightarrow W_{p}^{h-k} \ldots \ldots \ldots 373$

10.1.1 Coefficients of Operators Mapping $W_{p}^{h}$ into $W_{p}^{h-k}$

as Multipliers .............................. 374

10.1.2 A Counterexample....................... 378

10.1.3 Operators with Coefficients Independent of Some Variables . . . . . . . . . . . . . . . . . . . . . . 379

10.1.4 Differential Operators on a Domain ............... 382

10.2 Essential Norm of a Differential Operator ............... 384

10.3 Fredholm Property of the Schrödinger Operator ..........386

10.4 Domination of Differential Operators in $\mathbb{R}^{n} \ldots \ldots \ldots \ldots \ldots 387$ 
11 Schrödinger Operator and $M\left(w_{2}^{1} \rightarrow w_{2}^{-1}\right) \ldots \ldots \ldots \ldots \ldots 391$

11.1 Introduction ....................................... 391

11.2 Characterization of $M\left(w_{2}^{1} \rightarrow w_{2}^{-1}\right)$ and the Schrödinger

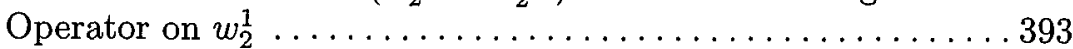

11.3 A Compactness Criterion ........................ 407

11.4 Characterization of $M\left(W_{2}^{1} \rightarrow W_{2}^{-1}\right) \ldots \ldots \ldots \ldots \ldots \ldots \ldots \ldots \ldots \ldots \ldots \ldots$

11.5 Characterization of the Space $M\left(\dot{w}_{2}^{1}(\Omega) \rightarrow w_{2}^{-1}(\Omega)\right) \ldots \ldots .416$

11.6 Second-Order Differential Operators Acting from $w_{2}^{1}$ to $w_{2}^{-1} \ldots 421$

12 Relativistic Schrödinger Operator

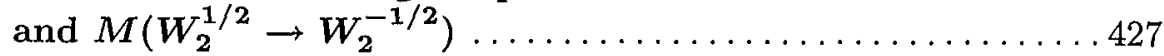

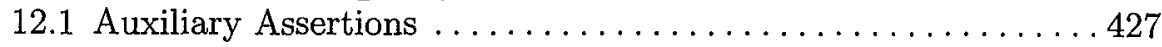

12.1.1 Main Result ............................ 436

12.2 Corollaries of the Form Boundedness Criterion and Related

Results.................................441

13 Multipliers as Solutions to Elliptic Equations ........... 445

13.1 The Dirichlet Problem for the Linear Second-Order Elliptic

Equation in the Space of Multipliers ................. 445

13.2 Bounded Solutions of Linear Elliptic Equations

as Multipliers ...............................447 44

13.2.1 Introduction $\ldots \ldots \ldots \ldots \ldots \ldots \ldots \ldots \ldots \ldots \ldots, \ldots \ldots \ldots$

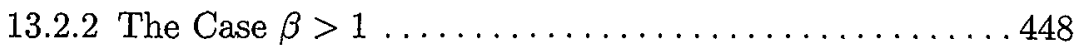

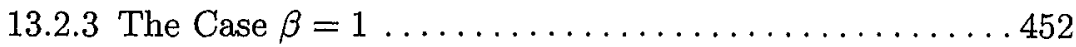

13.2.4 Solutions as Multipliers from $W_{2, w(\rho)}^{1}(\Omega)$ into $W_{2,1}^{1}(\Omega) .454$

13.3 Solvability of Quasilinear Elliptic Equations in Spaces

of Multipliers ............................ 456

13.3.1 Scalar Equations in Divergence Form . . . . . . . . 457

13.3.2 Systems in Divergence Form ................ 458

13.3.3 Dirichlet Problem for Quasilinear Equations

in Divergence Form .....................461

13.3.4 Dirichlet Problem for Quasilinear Equations

in Nondivergence Form................... 463

13.4 Coercive Estimates for Solutions of Elliptic equations

in Spaces of Multipliers ....................... 467

13.4.1 The Case of Operators in $\mathbb{R}^{n} \ldots \ldots \ldots \ldots \ldots \ldots \ldots 67$

13.4.2 Boundary Value Problem in a Half-Space . . . . . . . . . 469

13.4.3 On the $L_{\infty}$-Norm in the Coercive Estimate ........ 473

13.5 Smoothness of Solutions to Higher Order Elliptic Semilinear

Systems ................................ 474

13.5.1 Composition Operator in Classes of Multipliers . . . . . 474

13.5.2 Improvement of Smoothness of Solutions to Elliptic

Semilinear Systems .................... 477 
14 Regularity of the Boundary in $L_{p}$-Theory of Elliptic Boundary Value Problems ........................ 479

14.1 Description of Results ........................ 479

14.2 Change of Variables in Differential Operators ........... 481

14.3 Fredholm Property of the Elliptic Boundary Value Problem ... 483

14.3.1 Boundaries in the Classes $M_{p}^{l-1 / p}, W_{p}^{l-1 / p}$,

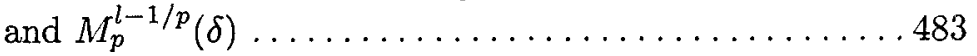

14.3.2 A Priori $L_{p}$-Estimate for Solutions and Other

Properties of the Elliptic Boundary Value Problem ... 484

14.4 Auxiliary Assertions . . ........................ 489

14.4.1 Some Properties of the Operator $\mathcal{T} \ldots \ldots \ldots \ldots \ldots \ldots 489$

14.4.2 Properties of the Mappings $\lambda$ and $\varkappa \ldots \ldots \ldots \ldots \ldots . \ldots 90$

14.4.3 Invariance of the Space $W_{p}^{l} \cap \mathscr{W}_{p}^{h}$ Under a Change

of Variables............................4492

14.4.4 The Space $W_{p}^{-k}$ for a Special Lipschitz Domain ...... 496

14.4.5 Auxiliary Assertions on Differential Operators

in Divergence Form . . . . . . . . . . . . . . . . . . . . 498

14.5 Solvability of the Dirichlet Problem in $W_{p}^{l}(\Omega) \ldots \ldots \ldots \ldots \ldots 502$

14.5.1 Generalized Formulation of the Dirichlet Problem .... . 502

14.5.2 A Priori Estimate for Solutions of the Generalized

Dirichlet Problem ......................502

14.5.3 Solvability of the Generalized Dirichlet Problem ...... . 503

14.5.4 The Dirichlet Problem Formulated in Terms of Traces . . 504

14.6 Necessity of Assumptions on the Domain .............. 507

14.6.1 A Domain Whose Boundary is in $M_{2}^{3 / 2} \cap C^{1}$ but does not Belong to $M_{2}^{3 / 2}(\delta) \ldots \ldots \ldots \ldots \ldots . \ldots 507$

14.6.2 Necessary Conditions for Solvability of the Dirichlet Problem..............................509

14.6.3 Boundaries of the Class $M_{p}^{l-1 / p}(\delta) \ldots \ldots \ldots \ldots \ldots \ldots \ldots 10$

14.7 Local Characterization of $M_{p}^{l-1 / p}(\delta) \ldots \ldots \ldots \ldots \ldots \ldots \ldots \ldots 13$

14.7.1 Estimates for a Cutoff Function .............. 513

14.7.2 Description of $M_{p}^{l-1 / p}(\delta)$ Involving a Cutoff Function . . 515

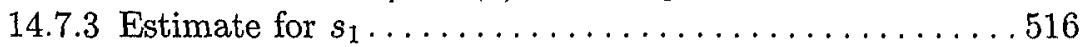

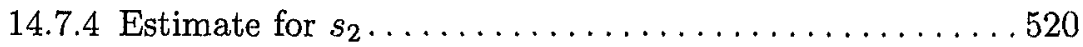

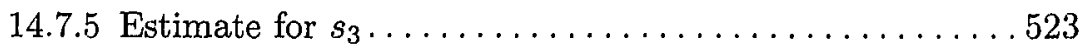

15 Multipliers in the Classical Layer Potential Theory for Lipschitz Domains . . . . . . . . . . . . . . . . . . . . 531

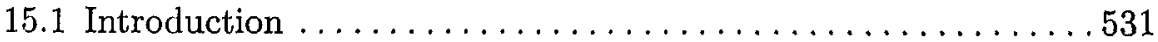

15.2 Solvability of Boundary Value Problems in Weighted Sobolev Spaces ................................ 537

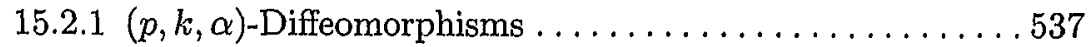

15.2.2 Weak Solvability of the Dirichlet Problem . . . . . . . . . 539

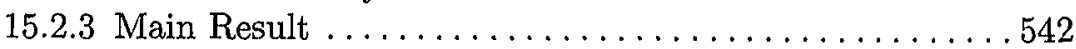


15.3 Continuity Properties of Boundary Integral Operators . . . . . 5 547

15.4 Proof of Theorems 15.1.1 and 15.1.2 .............. 551

15.4.1 Proof of Theorem 15.1.1................. 551

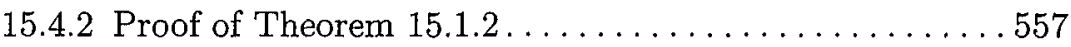

15.5 Properties of Surfaces in the Class $M_{p}^{\ell}(\delta) \ldots \ldots \ldots \ldots \ldots \ldots 55$

15.6 Sharpness of Conditions Imposed on $\partial \Omega \ldots \ldots \ldots \ldots \ldots \ldots 52$

15.6.1 Necessity of the Inclusion $\partial \Omega \in W_{p}^{\ell}$

in Theorem $15.2 .1 \ldots \ldots \ldots \ldots \ldots \ldots \ldots \ldots \ldots . \ldots \ldots 62$

15.6.2 Sharpness of the Condition $\partial \Omega \in B_{\infty, p}^{\ell} \ldots \ldots \ldots \ldots 563$

15.6.3 Sharpness of the Condition $\partial \Omega \in M_{p}^{\ell}(\delta)$

in Theorem $15.2 .1 \ldots \ldots \ldots \ldots \ldots \ldots \ldots \ldots 4 \ldots \ldots \ldots$

15.6.4 Sharpness of the Condition $\partial \Omega \in M_{p}^{\ell}(\delta)$

in Theorem 15.1.1 .................... 566

15.7 Extension to Boundary Integral Equations of Elasticity ...... 568

16 Applications of Multipliers to the Theory of Integral

Operators ............................... 573

16.1 Convolution Operator in Weighted $L_{2}$-Spaces .......... 573

16.2 Calculus of Singular Integral Operators with Symbols in Spaces of Multipliers ........................ 575

16.3 Continuity in Sobolev Spaces of Singular Integral Operators with Symbols Depending on $x \ldots \ldots \ldots \ldots \ldots \ldots \ldots \ldots \ldots$

16.3.1 Function Spaces ....................... 580

16.3.2 Description of the Space $M\left(H^{m, \mu} \rightarrow H^{l, \mu}\right) \ldots \ldots \ldots 582$

16.3.3 Main Result ............................. 585

16.3.4 Corollaries....................... 588

References.................................... 591

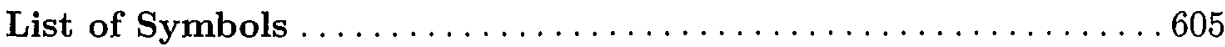

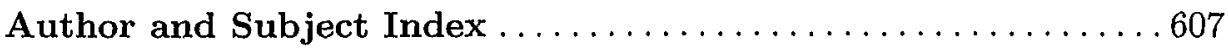

